\title{
Development of potent promoters that drive the efficient expression of genes in apple protoplasts
}

\author{
Xianpu Wang ${ }^{1}$, Lili Xu', Xiuxia Liu', Li Xin ${ }^{1}$, Shujing Wu $\mathbb{1}^{1 凶}$ and Xuesen Chen ${ }^{1 凶}$
}

\begin{abstract}
Protoplast transient expression is a powerful strategy for gene functional characterization, especially in biochemical mechanism studies. We herein developed a highly efficient transient expression system for apple protoplasts. The abilities of the Arabidopsis thaliana and Malus domestica ubiquitin-10 (AtUBQ10 and MdUBQ10) promoters to drive the expression of multiple genes were compared with that of the CaMV 355 promoter, and the results revealed that the AtUBQ10 and MdUBQ10 promoters were more efficient in apple protoplasts. With this system, we demonstrated that active AtMKK 7ac could activate MAPK6/3/4 signaling cascades, which further regulated MdWRKY33 phosphorylation and stability in apple. Furthermore, the ligand-induced interaction between the immune receptor AtFLS2 and the coreceptor AtBAK1 was reconstituted in apple protoplasts. We also found that the stability of the bacterial effector AvrRpt2 was regulated by feedback involving auxin and the immune regulator RIN4. The system established herein will serve as a useful tool for the molecular and biochemical analyses of apple genes.
\end{abstract}

\section{Introduction}

Apple is a rosaceous fruit tree plant that is cultivated worldwide, and researchers are becoming increasingly interested in the identification of the functionally important genes and molecular mechanisms involved in controlling apple fruit and plant growth and development ${ }^{1-3}$, fruit quality ${ }^{4-8}$, resistance ${ }^{9-12}$, and other physiological activities ${ }^{13,14}$. The expression of genes in plant materials is of critical importance for gene functional characterization and signal transduction pathway identification. Currently, apple genes are transiently expressed in onion epidermal cells $^{14}$, apple and maize protoplasts ${ }^{7,8,13,14}$, pollen tubes ${ }^{13}$, tobacco and apple leaves ${ }^{4,10}$ and apple fruit surface cells ${ }^{5}$ and are stably expressed in transgenic apple plants ${ }^{3,7,12}$, callus $^{6-8,11}$ and Arabidopsis ${ }^{8}$ for gene functional characterization. A protocol for transient expression in apple fruit cells was also developed by Spolaore et al. ${ }^{15}$. All these methods contribute to elucidating the molecular mechanisms controlling the physiological processes in apple.

\footnotetext{
Correspondence: Shujing Wu (shujingwu@sdau.edu.cn) or

Xuesen Chen (chanyetixi@163.com)

${ }^{1}$ College of Horticultural Science and Engineering, State Key Laboratory of

Crop Biology, Shandong Agricultural University, Tai'an, Shandong, PR China
}

Protoplasts are cell wall-free cells isolated from plant tissues and are capable of perceiving external stimuli. Transient expression in protoplasts has been proven to be a powerful strategy for gene functional characterization and signal pathway identification ${ }^{16}$, as it provides valuable information for understanding the molecular mechanisms controlling plant immune responses, hormone signaling, growth and development, epigenetic gene expression regulation and other physiological processes ${ }^{17-20}$. The expression of target genes in protoplast cells can be suppressed by the overexpression of artificial microRNAs or by modulation of their regulatory elements ${ }^{20,21}$. Mutagenesis in protoplast cells can be performed by CRISPR/Cas9 gene editing ${ }^{22,23}$. Protoplast transient expression has been found to efficiently, precisely, accurately, and consistently reveal molecular mechanisms. In apple, the use of an apple protoplast transient expression strategy is of special and critical importance. It can realize homologous expression without technical obstacles or the long time required to obtain transgenic apple plants and callus. Importantly, it is unaffected by the problems of heterologous expression. For example, certain Arabidopsis proteins were mislocalized after their expression in 
tobacco ${ }^{24}$. A precisely controlled expression strategy with an accurate evaluation method will be a powerful tool for apple gene functional characterization and signal transduction pathway identification, which will substantially deepen the understanding of the gene functions and signaling processes involved. In a recent report, apple protoplasts were subjected to CRISPR/Cas9 modification to generate genome-edited apple plants ${ }^{23}$. Despite the application of this method in several studies on subcellular localization and protein interactions ${ }^{7,8,13}$, it has not been extensively employed in studies on apple gene functional characterization and signal transduction pathway identification, especially in biochemical mechanism studies. Low gene expression in apple protoplast cells might be an important factor impeding the use of apple protoplasts.

In this study, target genes were stably and efficiently expressed in apple protoplasts by using the AtUBQ10 or $M d U B Q 10$ promoter. The data obtained herein using these promoters provide insight into the novel mechanisms regulating immune and disease resistance in apple. This research paves the way for the application of an apple protoplast transient expression strategy to elucidate biochemical mechanisms in the rosaceous plants.

\section{Results and discussion}

Intact apple protoplast cells that were spherical in shape and did not exhibit bursting or clustering were isolated from 'Orin' and 'Zihong' apple callus cells cultured in MS medium for different durations (Fig. 1a). The highest protoplast amounts were obtained from both 'Orin' and 'Zihong' apple callus cells cultured in MS medium for 10 days, yielding $3.68 \times 10^{6}$ and $4.07 \times 10^{6}$ protoplasts per gram of fresh weight callus (per g FW callus), respectively (Fig. 1b). Although apple callus cells cultured in MS medium for 6 days yielded only $1.3 \times 10^{6}$ protoplasts per $\mathrm{g}$ FW, this condition yielded the strongest target protein expression (Supplementary File 1: Fig. S3a) and was therefore used for protoplast transfection in our research.

\section{Pro-BIUTNT is a potent promoter that enables the efficient expression of target genes in apple protoplast cells}

Ethylene response factors (ERFs) are a large family of transcription factors that are extensively involved in controlling fruit ripening, disease resistance and other physiological activities or traits. MdERF1 and MdERF2 are two ERF transcription factors involved in controlling fruit ripening ${ }^{25}$. In our apple protoplast transient expression system driven by the cauliflower mosaic virus (CaMV) $35 \mathrm{~S}$ promoter, MdERF1 expression was not detectable, and a weak MdERF2 signal was detected by western blot. However, a clear and specific MdERF1 signal and a strong MdERF2 signal were detected when the promoter of Arabidopsis ubiquitin-ten (Pro-BIUTNT) was utilized
(Fig. 2b), which is the 1307 nucleotide sequence upstream of the ATG translational start codon of the Arabidopsis ubiquitin-10 (At4g05320) gene. Further studies revealed that two of the three independent MdERF2 clones under the control of CaMV $35 S$ generated a dramatically weaker signal than that generated using Pro-BIUTNT (Fig. 2c). Only trace amounts of MdERF1 were detected in two MdERF1 clones under the control of CaMV $35 S$ compared with the significantly stronger and more specific signal of the gene driven by Pro-BIUTNT (Fig. 2c).

MdERF3 and MdERF6 are two ERF transcription factors that are responsive to pathogen infection ${ }^{26}$. Homologs of MdERF3 and MdERF6, MdERF3a and MdERF6a were cloned and transfected into apple protoplast cells. Strong MdERF3a and MdERF6a signals were detected when the genes were driven by Pro-BIUTNT, but little or no signal was detected when the genes were driven by $C a M V 35 S$ (Fig. 2c).

ERF98 (At3g23230) is an ERF transcription factor that is responsive to treatment with flg22, a conserved 22-amino acid peptide from eubacterial flagellin that induces immune responses in plants ${ }^{27}$, and MdERF98 is the ortholog of ERF98 in the apple genome. No expression was found when driven by $C a M V 35 S$, but a strong signal was detected for MdERF98 driven by Pro-BIUTNT (Fig. 2b). Analysis of a different $C a M V$ 35S::MdERF98 clone also revealed that the gene under the control of $C a M V$ $35 S$ was expressed at lower levels in protoplast cells than the gene driven by the Pro-BIUTNT promoter (Supplementary File 1: Fig. S1). This result suggests that CaMV $35 S$ was not suitable for driving the expression of the target genes in apple protoplast cells and was consistent with the expression of three independent MdERF2 and MdERF1 clones driven by the CaMV $35 S$ promoter in further assays.

MdEIL2 is the ortholog of EIN3 and regulates the expression of $M d P G 1^{28}$. A strong and clear MdEIL2 signal was achieved using Pro-BIUTNT, in contrast to the weak signal achieved with CaMV $35 S$ (Fig. 2d).

WRKY33 and WRKY29 are involved in regulating ethylene and phytoalexin biosynthesis and pathogenassociated molecular pattern (PAMP)-triggered immune responses, respectively ${ }^{29,30}$. The orthologs of the two genes in apple, MdWRKY33 and MdWRKY29 (Table S1), were cloned and transfected into apple protoplast cells. MdWKY33 was hardly expressed under the control of CaMV 35S; however, a strong signal was obtained when it was driven by Pro-BIUTNT (Fig. 2b, Supplementary File 1: Fig. S3a, b). To further explore and compare the capabilities of Pro-BIUTNT and CaMV $35 S$ to drive the expression of target genes in apple protoplast cells, the C-terminal epitope HA tag of MdWRKY33 was replaced with GFP. Strong MdWRKY33-GFP expression was detected when the gene was driven by Pro-BIUTNT, while 
a
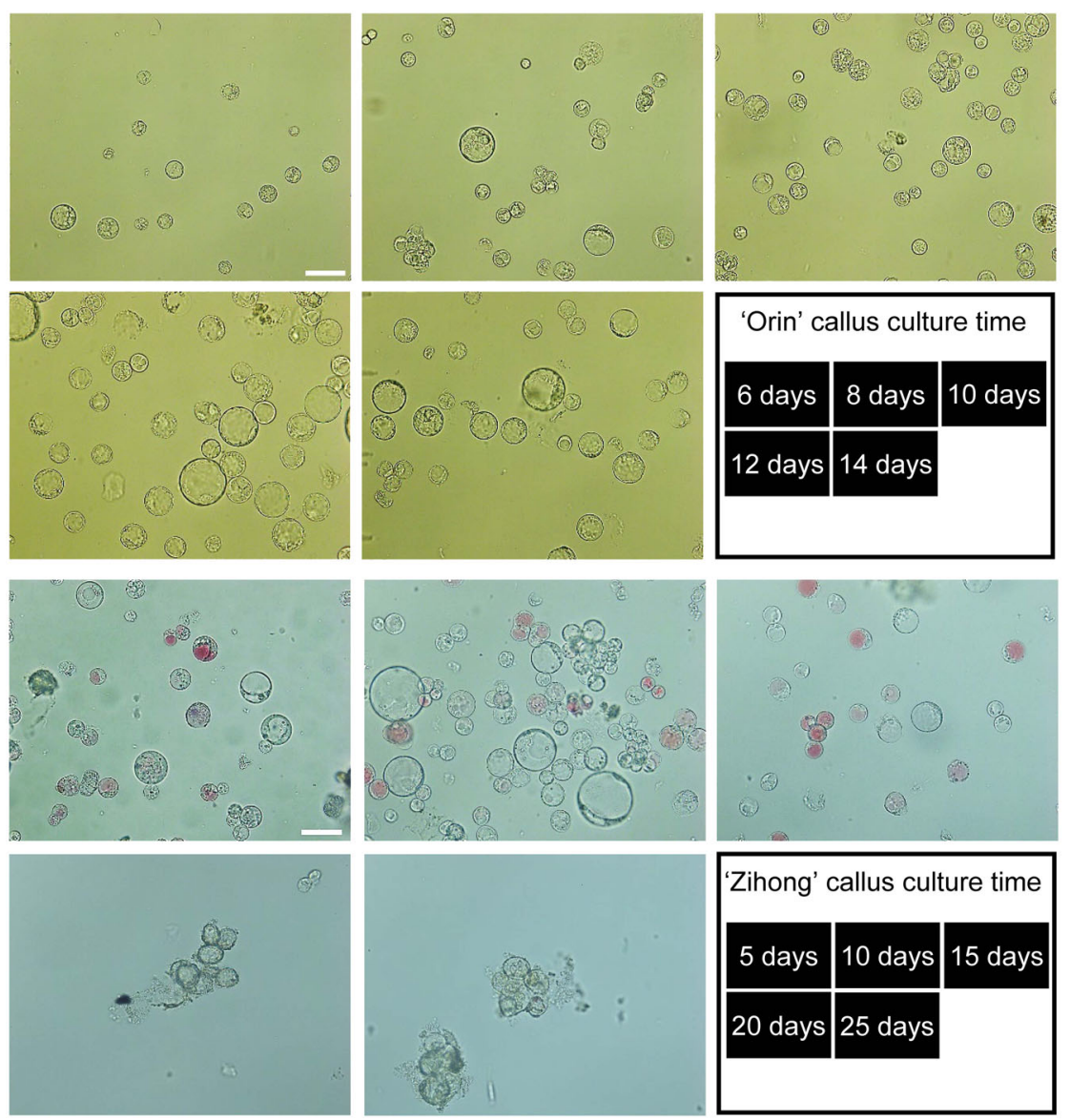

b

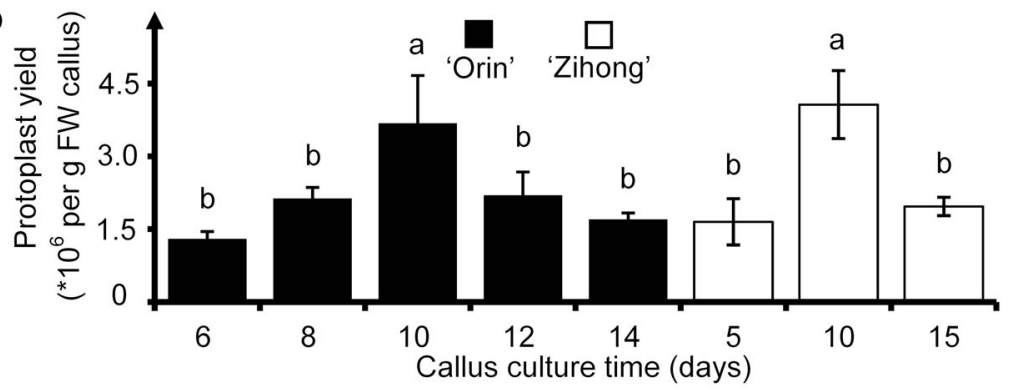

C

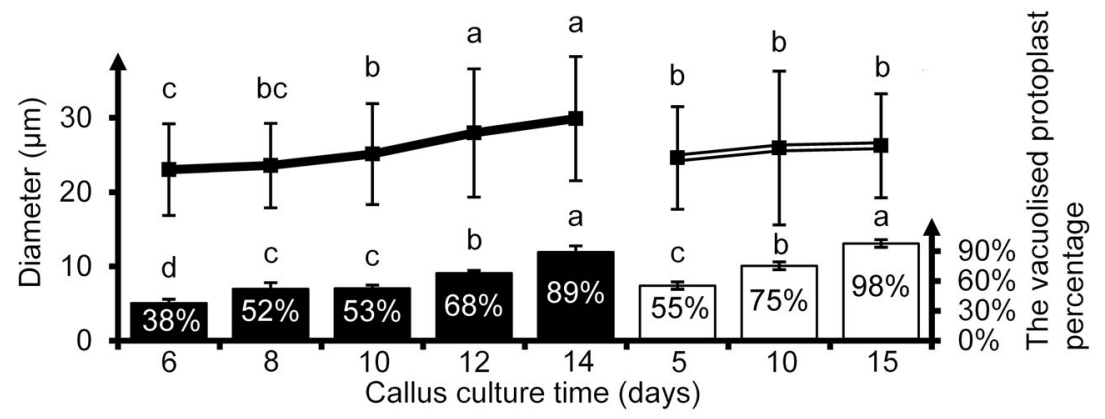

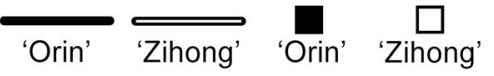

Fig. 1 (See legend on next page.) 
(see figure on previous page)

Fig. 1 Protoplast cells isolated from apple callus cells cultured in MS medium for different durations. a 'Orin' (upper panel) and 'Zihong' (lower panel) apple protoplast cells under microscopy. The culture times of the apple callus cells used for protoplast isolation are shown to the right of the lower row in each panel. The scale bars represent $50 \mu \mathrm{m}$. b Protoplast yield of 'Orin' (filled columns) and 'Zihong' callus cells (open columns). FW fresh weight. c Average diameters (polyline) and percentage ratios of vacuolated cells (columns) in 'Orin' (filled symbols) and 'Zihong' (open symbols) protoplast cells $(n \geq 100)$. One-way ANOVA and multiple comparisons via Fisher's LSD test were performed $(p<0.05)$. Different characters at the top of the columns indicate significant differences. Three independent experiments were conducted, and one representative result is shown
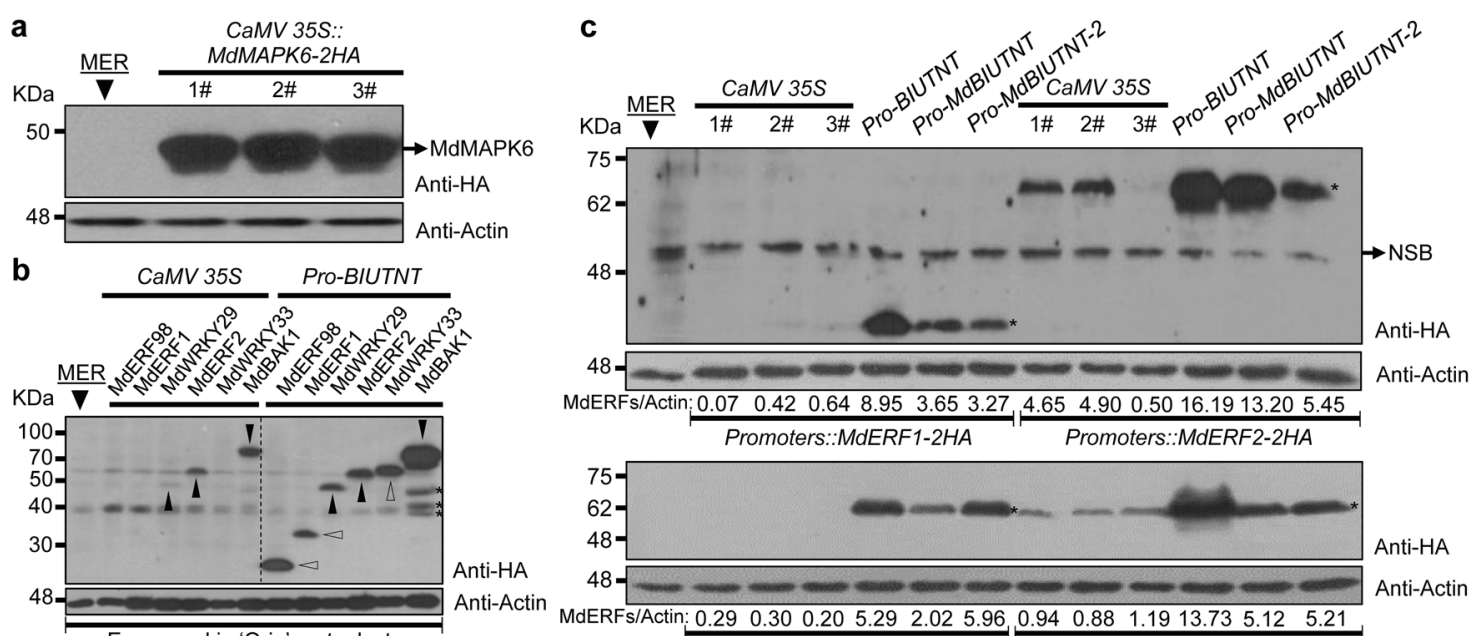

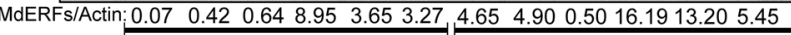
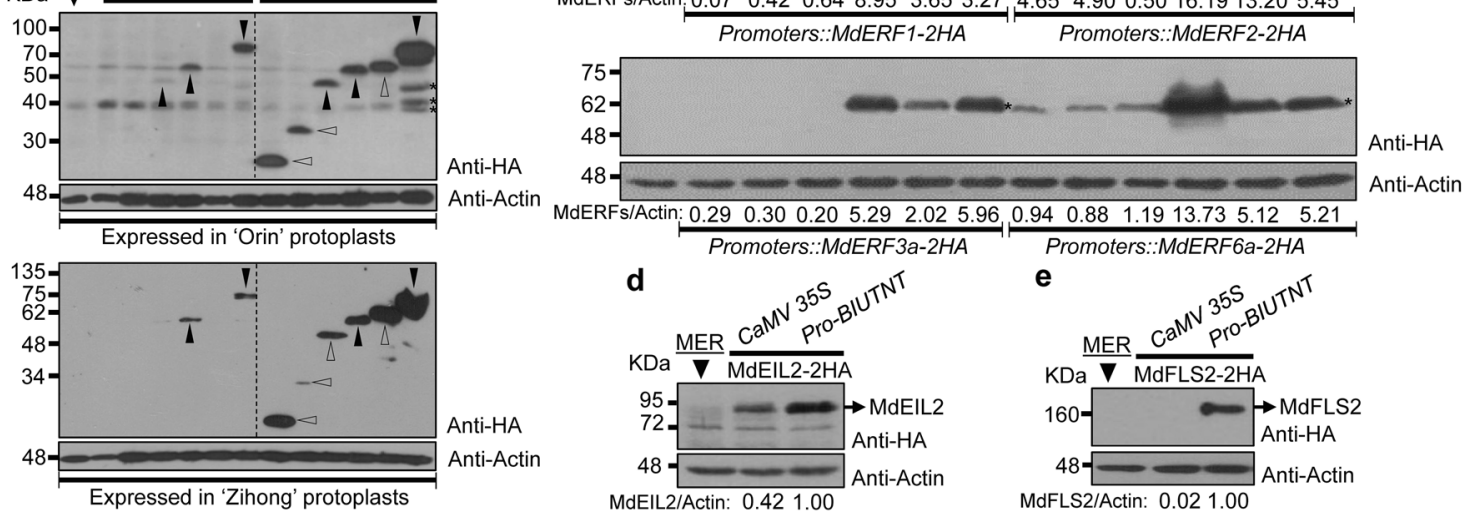

Fig. 2 Stable and efficient expression of the tested genes in apple protoplast cells driven by AtUBQ10 (At4g05320) or MdUBQ10 (MDP0000820500) promoters. a MdMAPK6 expression driven by CaMV 35S. The expression of three independent clones is shown. $\mathbf{b}$ The expression of the tested genes in apple protoplast cells driven by CaMV $35 \mathrm{~S}$ and Pro-BIUTNT. Filled and open triangles indicate weaker or stronger signals and the absence or presence of signals driven by CaMV $35 S$ and Pro-BIUTNT, respectively. The asterisks indicate three processed bands of MdBAK1. c Further comparison of the abilities of the CaMV 35 S and UBQ10 promoters to drive the expression of the tested genes in apple protoplast cells. Three independent clones of the expression vector using CaMV 355 were selected. Asterisks indicate the signals of the expressed proteins. NSB nonspecific band. Pro-BIUTNT, the 1307-base pair (bp) sequence upstream from the AtUBQ10 ATG translational start codon; Pro-MdBIUTNT, the 1539bp sequence upstream from the MdUBQ10 ATG translational start codon; Pro-MdBIUTNT-2, the 2501-bp sequence upstream from the MdUBQ10 ATG translational start codon. d MdEIL2 expression driven by the CaMV $35 S$ and Pro-BIUTNT promoters. e MdFLS2 expression driven by the CaMV $35 S$ and Pro-BIUTNT promoters. The intensities of the expressed proteins relative to that of actin are shown in $\mathbf{c}-\mathbf{e}$. The signal intensity was calculated by ImageJ. Actin was used as the internal control. MER represents a control sample transfected with the expression vector in which the target genes were replaced with a meaningless random sequence

no expression was found under the control of $\mathrm{CaMV} 35 \mathrm{~S}$ (Supplementary File 1: Fig. S4a). CaMV $35 S$ has numerous variants, and the variant we herein named $C a M V 35 S$ 2 was subcloned from the pBI121 binary vector ${ }^{31}$ and analyzed for its ability to drive the expression of the target genes. Both of the CaMV $35 S$ promoters contained the core sequence of the CaMV $35 \mathrm{~S}$ promoter (Supplementary File 1: Sequences 1 and 2), and their abilities to drive the expression of target genes in apple have been proven ${ }^{11}$ (Fig. 4f). This variant of $C a M V 35 S$ resulted in weak
MdERF98 expression and no MdWRKY33 expression (Supplementary File 1: Fig. S4b, c).

The above results suggested that Pro-BIUTNT is a strong promoter that enables the stable expression of target genes in apple protoplast cells.

Previous research showed that the AtUBQ10 promoter was more stable and persistent than CaMV $35 \mathrm{~S}$ in Arabidopsis and tobacco ${ }^{32}$, and the target gene was expressed at higher levels under the control of the AtUBQ10 promoter in both dicot (Arabidopsis) and monocot (rice) 


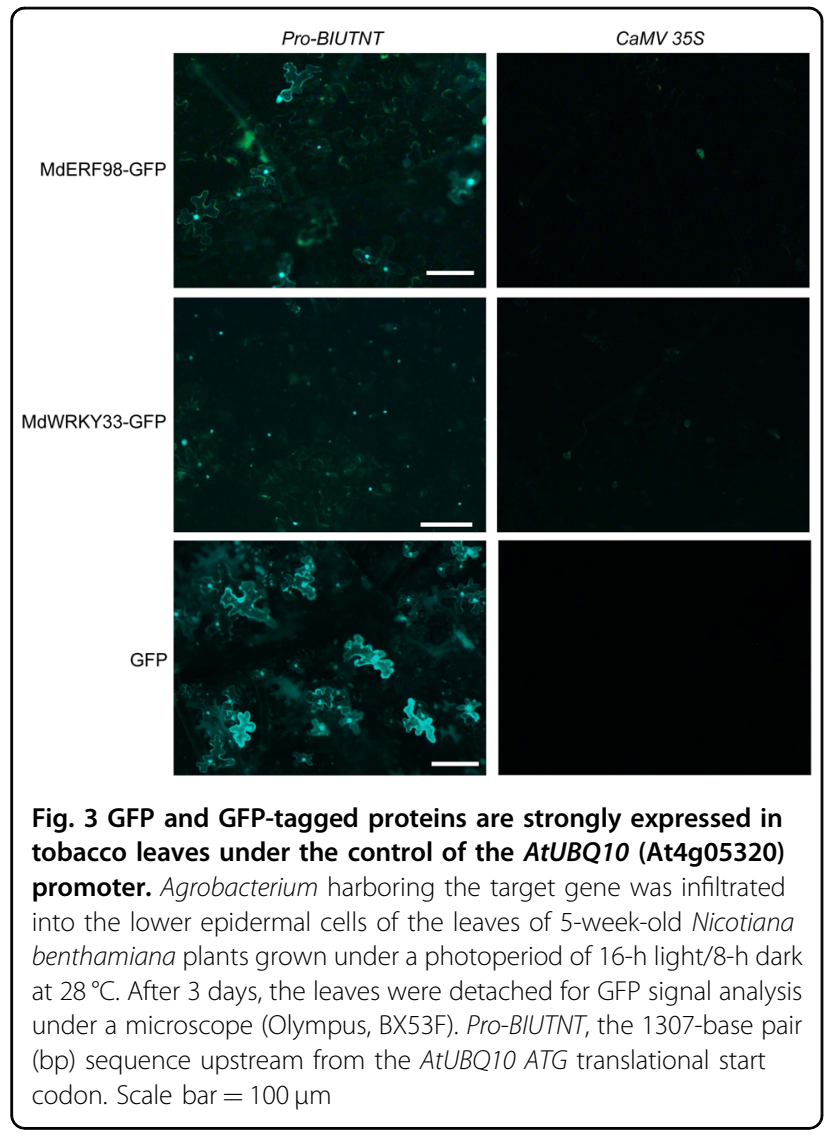

plants $^{33}$. Stronger WRKY33-GFP, MdERF98-GFP, and GFP expression in tobacco leaf epidermal cells was observed when they were driven by Pro-BIUTNT than when they were driven by $C a M V 35 S$ (Fig. 3), which was consistent with the stronger activity of the AtUBQ10 promoter in apple protoplast cells (Fig. 2). Gene silencing contributes to the low expression of target genes driven by $C a M V 35 S$ in transgenic plants ${ }^{33}$. In apple, the expression level of the scab resistance gene $H c r V f 2$ under the control of CaMV $35 S$ was 100 -fold lower than that driven by the native promoter ${ }^{12}$. Whether gene silencing contributes to the low expression under the control of $C a M V 35 S$ in apple plants needs to be investigated further. However, the strong activity of Pro-BIUTNT and the contrasting low activity of CaMV $35 S$ may be determined predominantly by the differences in the genetic backgrounds of apple and other plants, such as Arabidopsis and cotton $^{16,17,34}$. CaMV $35 S$ and Pro-BIUTNT showed similar activity levels in Arabidopsis, and while their activities were significantly reduced in apple protoplast cells, the activity of Pro-BIUTNT was $\sim 5$ - to 10-fold higher than that of CaMV 35S (Supplementary File 1: Figs. S6, S2b). Another finding supporting our conclusion is that the promoter of MdUBQ10 (MDP0000820500), the ortholog of $A t U B Q 10$ in the apple genome, drove the expression of target genes in apple protoplast cells significantly better than CaMV $35 S$ (Fig. 2c). Notably, the MdUBQ10 promoter sequence was not highly similar to that of ProBIUTNT (Supplementary File 1: Table S2).

Our research demonstrated that the Pro-BIUTNT and $M d U B Q 10$ promoter enable the efficient expression of target genes in apple protoplast cells.

\section{AtMKK7ac overexpression activates MAPK signaling in apple}

MAPK signaling is an ancient and conserved signaling pathway in eukaryotes ${ }^{35}$ that helps to control plant growth, development, and disease resistance ${ }^{30,35}$. However, its upstream activators have not been identified in apple.

AtMKK7 (At1g18350) is thought to be involved in regulating both basal and systemic acquired resistance in plants $^{36}$. The constitutive expression of AtMKK7 in its active form (AtMKK7ac: AtMKK7 with S193ES199D mutation) induces hypersensitive reactions in tobacco leaves (Fig. 4c). The in vivo detection of MdMAPK phosphorylation using an anti-pERK antibody showed that the constitutive expression of AtMKK7ac in apple protoplast cells markedly activated the phosphorylation of MdMAPK3/6/4, particularly MdMAPK4 (Fig. 4d). AtMKK7ac was then expressed in apple callus cells via Agrobacterium-mediated transient expression, and AtMKK7ac overexpression strongly activated MdMAPK phosphorylation (Fig. 4g). An in vitro kinase assay showed that MBP-AtMKK7ac could phosphorylate MdMAPK6, and Thr-231 was important for this process (Fig. 4f).

In this study, apple protoplasts were isolated from genetically transformed 'Orin' apple callus cells expressing MdMAPK6-FLAG driven by CaMV $35 S$ and from wildtype 'Orin' apple callus cells. The coexpression of AtMKK7ac and MdWRKY33 induced a band shift of MdWRKY33 in apple protoplast cells overexpressing MdMAPK6-FLAG, which mimicked the band shift of Botrytis-induced kinase 1 (BIK1) observed in PAMPtriggered immune responses ${ }^{37}$, whereas a similar band shift was not observed in protoplast cells expressing only MdWRKY33. This result suggested that the expression of MdWRKY33 in apple protoplast cells was modified, potentially by phosphorylation, by upstream activators. The level of MdWRKY33 in the presence of MdMAPK6FLAG was markedly higher than that obtained without MdMAPK6-FLAG overexpression (Fig. 4e), which suggests that the stability of MdWRKY33 is influenced by the status and degree of its phosphorylation upon activation by upstream factors.

The revelation that AtMKK7ac activates MdMAPK6 provides an effective method for activating MAPK signaling in apple cells and for elucidating the elements controlling MdMAPK6 activation in apple. 


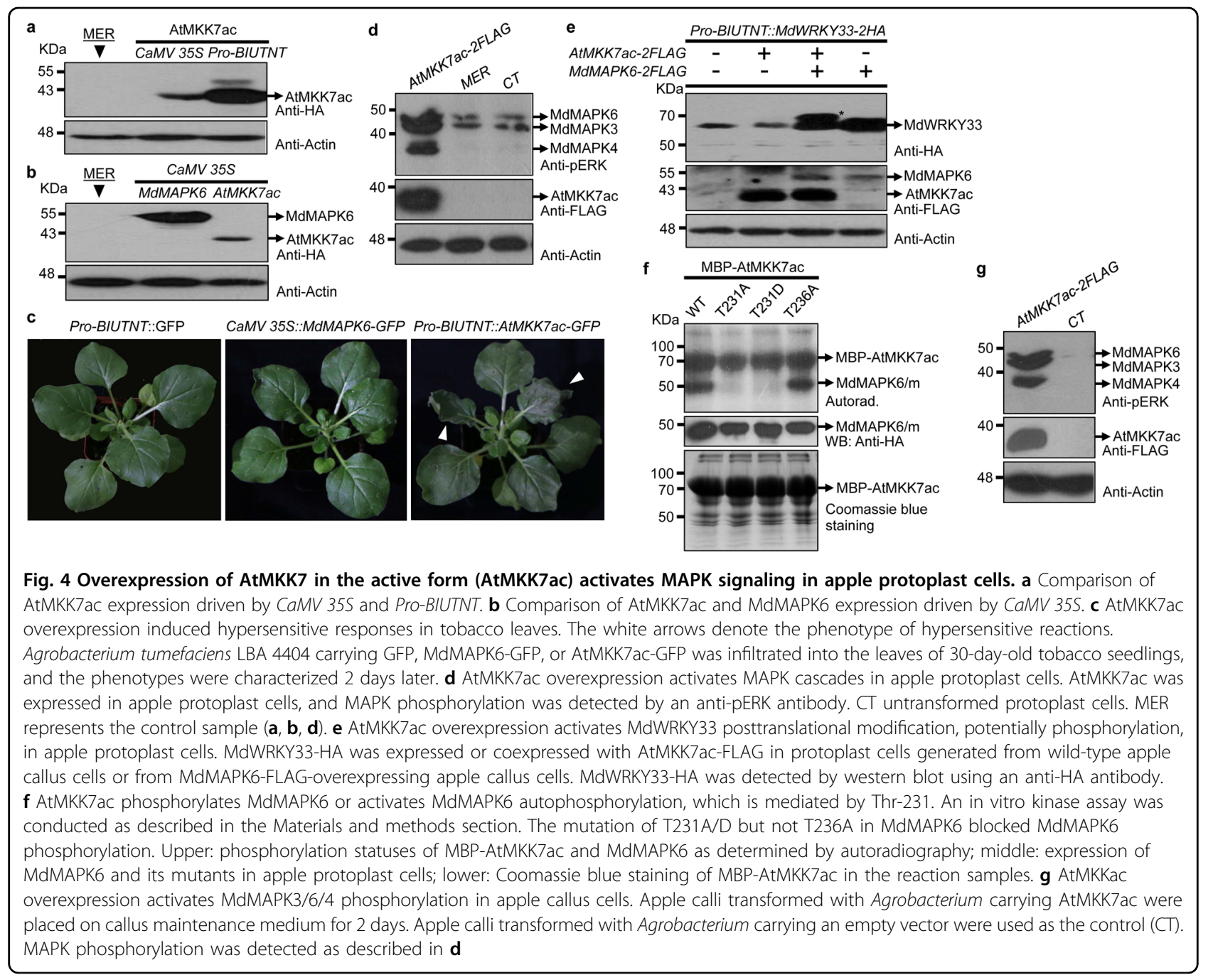

Pro-BIUTNT enables the reconstitution of BAK1 and FLS2 interaction in apple protoplast cells

The interaction of AtBAK1 and AtFLS2 is an important and fundamental signaling event in plant immune responses triggered by $\mathrm{PAMPs}^{38}$. Immune complex formation was observed in apple protoplast cells after flg22 elicitation. In this study, AtFLS2 with a C-terminal epitope tag FLAG (AtFLS2-FLAG) and AtBAK1 with an HA tag (AtBAK1-HA) were coexpressed in apple protoplast cells driven by Pro-BIUTNT. A coimmunoprecipitation assay was then performed to investigate the interaction between the two proteins in protoplasts. The AtBAK1HA signal was found in the AtFLS2-FLAG complex immunoprecipitated from apple protoplast cells treated with flg22, whereas no signal was detected in AtFLS2FLAG complexes immunoprecipitated from apple protoplasts that were not subjected to flg22 elicitation or from protoplasts expressing only MdBAK1-2HA (Fig. 5).
This study elucidated the paradigmatic signaling event in apple protoplast cells and provides a direct method for identifying novel signaling components of PAMPtriggered immune responses in highly developed perennial woody plants. This investigation also provides an accurate and convenient method for comparative studies of immune signaling in model and highly developed plants.

\section{Feedback regulation of AvrRpt2 by auxin and the RIN4- associated complex}

AvrRpt2, a type III effector from Pseudomonas syringae, promotes pathogen virulence by enhancing auxin signaling and activates disease resistance by cleaving RIN4 in plants ${ }^{39,40}$. We previously showed that AvrRpt2 enhances auxin signaling by accelerating the turnover of the auxin signaling transcriptional repressor $\mathrm{AXR} 2{ }^{40}$. 


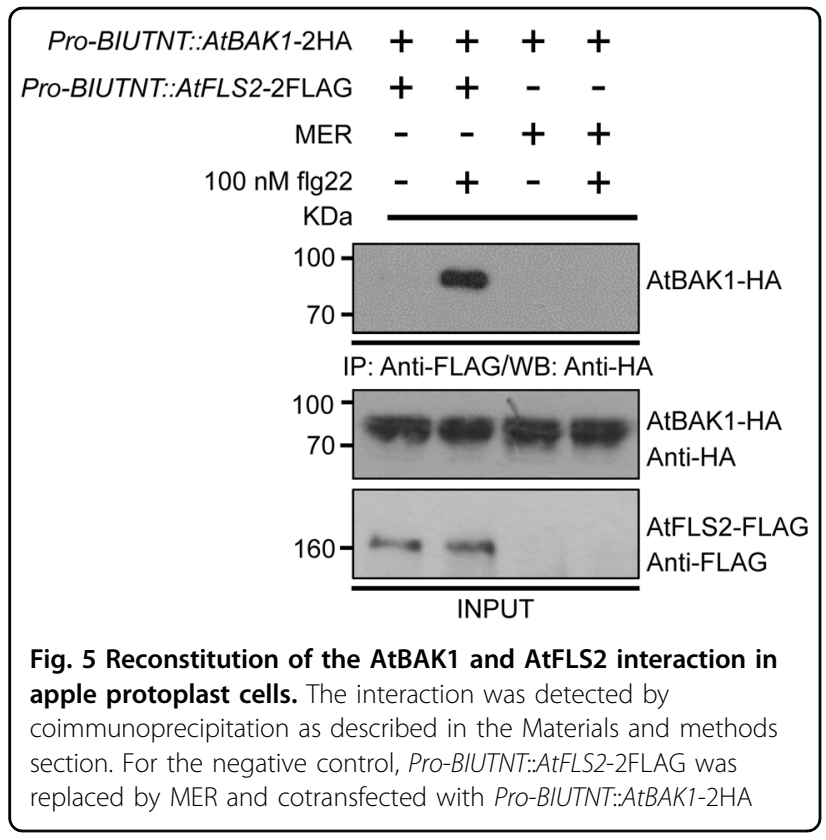

Consistent with previous findings, AXR2 expression was significantly reduced in the presence of AvrRpt2 and 1-naphthalacetic acid (NAA), and the P87S mutation in AXR2 blocked NAA- and AvrRpt2-mediated degradation (Fig. 6b). Interestingly, the protein level of AvrRpt2 was markedly decreased to undetectable levels in the presence of $1 \mathrm{mM}$ NAA (Fig. $6 \mathrm{~b}$ ). The same results were found in tobacco leaves when AvrRpt2 was expressed or coexpressed together with AXR2 or AXR2 P87S (Fig. 6c).

The degradation of AvrRpt2 was also identified in its interactions with RIN4. The functional processing of RIN4 by AvrRpt 2 was confirmed by the disappearance of full-length RIN4-GFP [indicated as RIN4 (FL) in Fig. 6d] and the detection of the GFP-tagged RIN4 C-terminus [indicated as RIN4 (AMC) in Fig. 6d] upon the coexpression of RIN4-GFP with AvrRpt2-HA. We also repeatedly found that the AvrRpt2 level was decreased or even undetectable in the presence of RIN4 (Fig. 6d, Supplementary File 1: Fig. S5a, b).

These results suggest that the microbial effector is negatively regulated by auxin and the RIN4-associated complex in the plant. This phenomenon might represent two important disease resistance mechanisms in plant basal and effector-triggered resistance. The strengthened auxin signaling might provide feedback to remove the virulence source inside cells and thereby enhance plant disease resistance. In AvrRpt2-activated disease resistance mediated by the nucleotide-binding domain leucine-rich repeat (NLR) protein RPS2, AvrRpt 2 might be negatively controlled to prevent the excessive induction of immune responses triggered by the causal agent itself. The detailed mechanisms by which auxin and the RIN4 complex

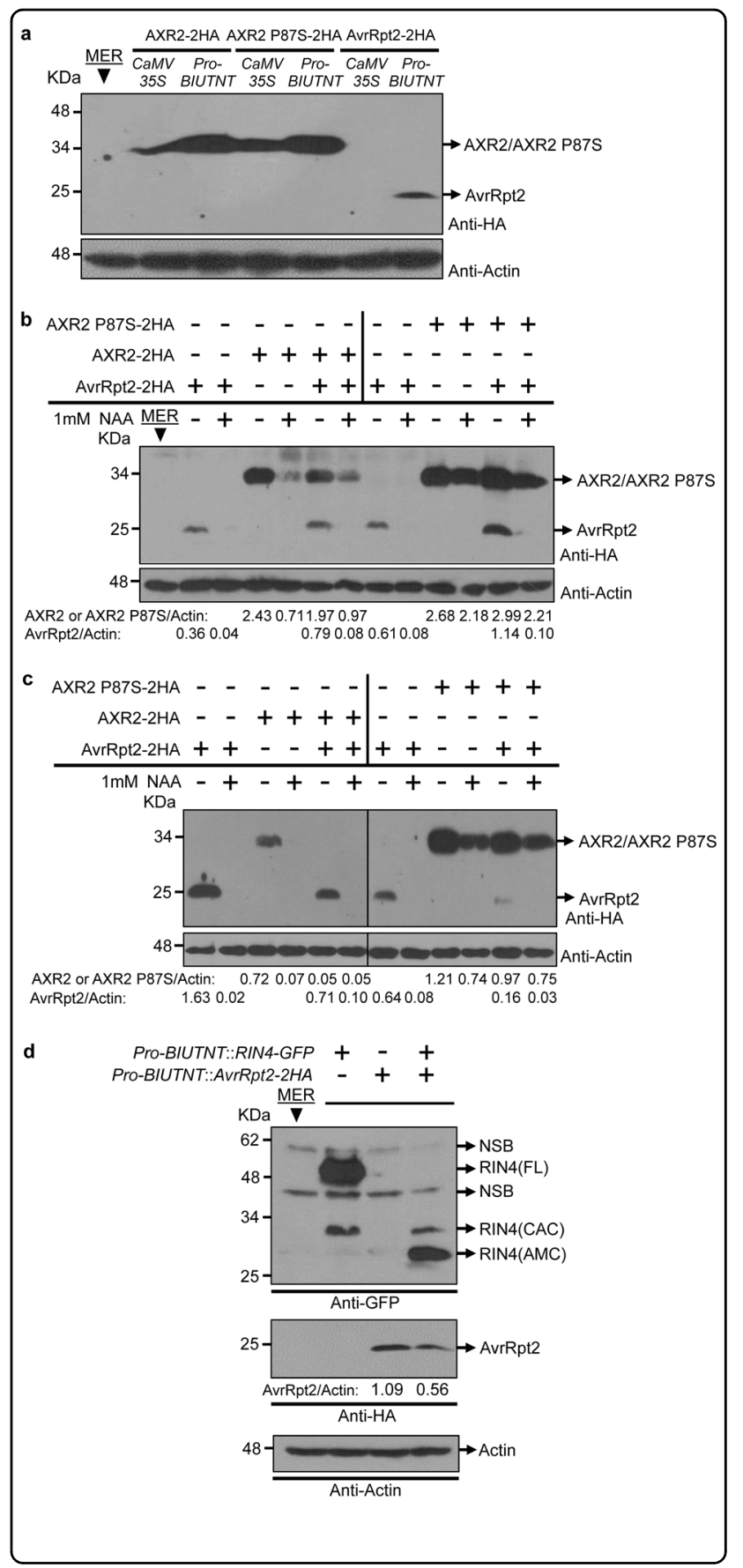

control the microbial effector in plant cells will be elucidated in the future.

\section{Materials and methods \\ Plasmid construction}

Pro-BIUTNT, an abbreviation for 'promoter of ubiquitin-ten', the 1307-base pair (bp) sequence upstream from the translational start codon ATG of Arabidopsis 
Fig. 6 Negative and feedback regulation of AvrRpt2 by auxin signaling and the RIN4-associated complex. a AXR2, AXR2 P87S, and AvrRpt2 expression in apple protoplasts driven by CaMV 355 or Pro-BIUTNT. Negative regulation of AvrRpt2 by auxin signaling. AXR2 or AXR2 P87S was expressed or coexpressed with AvrRpt2 in apple protoplast cells (b) or tobacco leaves (c). The transfected protoplasts were incubated for $4 \mathrm{~h}$ and then treated with $1 \mathrm{mM}$ NAA for another $2 \mathrm{~h}$. AXR2 or AXR2 P87S was also expressed or coexpressed with AvrRpt2 in tobacco leaves for 3 days and then collected for treatment with $1 \mathrm{mM}$ NAA for $6 \mathrm{~h}$. The accumulation of proteins was detected by western blot using an anti-HA antibody. The signal intensities were calculated by ImageJ. The intensities of AXR2, AXR2 P87S, and AvrRpt2 relative to that of actin are shown beneath the actin signal. $\mathbf{d}$ Negative regulation of AvrRpt2 by the RIN4-associated complex. C-terminal epitope-tagged RIN4 with GFP (RIN4-GFP) and AvrRpt2 tagged with HA (AvrRpt2-HA) were coexpressed, driven by Pro-BIUTNT, in apple protoplast cells for $6 \mathrm{~h}$. Protoplasts expressing only RIN4-GFP or AvrRpt2-HA were used as controls. Western blot analysis was conducted to detect the accumulation of the expressed proteins using anti-GFP (upper panel) or anti-HA antibodies (middle panel). Actin was used as the internal standard. The intensity of AvrRpt2-HA relative to that of actin (AvrRpt2/Actin) is shown. MER was used as the negative control. The experiment was repeated three times, and one representative result is shown. RIN4 (CAC) cell autologous cleavagereleased band of RIN4, RIN4 (AMC) GFP-tagged C-terminus of RIN4 released by AvrRpt2-mediated cleavage, RIN4 (FL) full-length version of RIN4, NSB nonspecific band

ubiquitin-10 (At4g05320), was amplified from Arabidopsis genomic DNA.

pHBT-CaMV 35S::AvrRpm1-2HA was used as the backbone for the construction of the protoplast transient expression vectors used in this study. The vector driven by CaMV $35 \mathrm{~S}$ was constructed by directly replacing AvrRpm1 with the coding sequence of the target gene. The vector driven by the Pro-BIUTNT or MdUBQ10 (MDP0000820500) promoter was constructed by substituting Pro-BIUTNT or nucleotide sequences of different lengths upstream from the $A T G$ translational start codon of MdUBQ10 for CaMV 35S.

\section{Protoplast isolation, transfection, and western blot analysis}

Apple protoplast cells were isolated from 'Orin' and 'Zihong' apple callus cells that had been cultured in MS medium for different durations according to the method described by Gao et al. ${ }^{34}$. One gram of apple callus cells was dispersed evenly in $10 \mathrm{~mL}$ of enzyme digestion solution consisting of $1.5 \%$ cellulase, $0.4 \%$ macerozyme, and $0.05 \%$ pectinase dissolved in $20 \mathrm{mM}$ MES buffer containing $20 \mathrm{mM} \mathrm{KCl}, 10 \mathrm{mM} \mathrm{CaCl}, 2 \%$ sucrose, and $0.4 \mathrm{M}$ mannitol ( $\mathrm{pH}$ adjusted to 5.7). After vacuum infiltration for $30 \mathrm{~min}$, the digestion solution was maintained undisturbed at room temperature for $8 \mathrm{~h}$. The solution was then passed through a nylon mesh with a diameter of $100 \mu \mathrm{m}$ and collected in a $50 \mathrm{~mL}$ Eppendorf tube, to which an equal volume of W5 solution was added. The nylon mesh was washed twice with W5 solution, and the solution was collected and added to the protoplast cell suspension. After the suspension was centrifuged at $650 \times$ $g$ for $2 \mathrm{~min}$, the protoplast cells were collected, resuspended in $15 \mathrm{~mL}$ of W5 solution, and maintained on ice for $30 \mathrm{~min}$. The supernatant was then discarded, and the protoplast cells were suspended in $1 \mathrm{~mL}$ of MMG solution and later used for protoplast cell number calculations and plasmid DNA transfection.

Protoplasts were transfected as described by He et al. ${ }^{16}$. Protein expression in protoplast cells was detected by western blot using anti-HA, anti-FLAG, or anti-GFP antibodies.

\section{Luciferase activity assay}

AvrRpm1 in pHBT-CaMV 35S::AvrRpm1-2HA was replaced with a luciferase (Luc) coding sequence to generate pHBT-CaMV 35S::Luc. CaMV $35 S$ was then replaced to generate luciferase vectors under the control of the promoters investigated in the research. The luciferase activity in apple or Arabidopsis protoplast cells was measured according to $\mathrm{He}$ et al. ${ }^{16}$.

\section{Co-immunoprecipitation (Co-IP)}

Driven by Pro-BIUTNT, AtBAK1-HA and AtFLS2FLAG were expressed in apple protoplast cells for $6 \mathrm{~h}$. After treatment with $100 \mathrm{nM}$ flg22 or the equivalent amount of solvent for an additional $10 \mathrm{~min}$, the protoplast cells were harvested. Co-IP was conducted using antiFLAG beads according to Lu et al. ${ }^{37}$.

Cells expressing AtBAK1 that were treated or not treated with only flg22 were used as controls.

\section{In vitro kinase assay}

MdMAPK6 (MDP0000340624) or its mutant MdMAPK6 T231A, MdMAPK6 T231D, or MdMAPK6 T236A was cloned into a pBI121 binary vector under the control of CaMV $35 S$ with a C-terminal FLAG epitope tag and transformed into apple callus cells according to $\mathrm{He}$ et al. ${ }^{11}$.

The recombinant protein MBP-AtMKK7ac was induced and purified from E. coli BL21.

In the kinase activity assay, MdMAPK6 or one of its mutants was purified from one gram of transgenic apple callus cells and mixed with two micrograms of MBPAtMKK7ac in $25 \mu \mathrm{L}$ of kinase reaction buffer $(20 \mathrm{mM}$ Tris- $\mathrm{HCl}$ [pH 7.5], $5 \mathrm{mM}$ EDTA [pH 8.0], $1 \mathrm{mM}$ DTT, $20 \mathrm{mM} \mathrm{MgCl}_{2}, 0.1 \mathrm{mM}$ ATP and $\left.5 \mu \mathrm{Ci}\left[\gamma^{-}{ }^{32} \mathrm{P}\right] \mathrm{ATP}\right)$. After reacting for $2 \mathrm{~h}$ at $23^{\circ} \mathrm{C}$, the proteins were denatured and separated by SDS-PAGE. Phosphorylation was detected by autoradiography.

\section{Acknowledgements}

This work was supported by grants from the National Key Research and Development Program of China (2018YFD1000307) and the Natural Science Foundation in China (nos. 31672136 and 31272132). We thank Professors Libo 
Shan and Ping He of Texas A\&M University for providing the CaMV 35S-driven protoplast transient expression vector and for their helpful comments and revisions regarding the manuscript. We thank Professor Yujin Hao of Shandong Agricultural University for providing the 'Orin' apple callus cells. We appreciate American Journal Experts (AJE) for editing the English language in the manuscript.

\section{Author contributions}

X.W., X.C., and S.W. conceived the project and designed the research; X.W., L.Xu., X.L., and L.X. performed all the experiments; X.W., X.C., and S.W. analyzed the experimental data and discussed the results; and X.W. and S.W. wrote the manuscript.

\section{Data availability}

All data supporting the findings of this study are available within the paper or within its supplementary information published online. The materials used in this study are available from the corresponding author upon reasonable request.

\section{Conflict of interest}

The authors declare no competing interests.

Supplementary information The online version contains supplementary material available at https://doi.org/10.1038/s41438-021-00646-4.

Received: 1 February 2021 Revised: 29 June 2021 Accepted: 5 July 2021 Published online: 01 October 2021

\section{References}

1. Yao, J. L., Dong, Y. H. \& Morris, B. A. M. Parthenocarpic apple fruit production conferred by transposon insertion mutations in a MADS-box transcription factor. Proc. Natl Acad. Sci. USA 98, 1306-1311 (2001).

2. Ireland, H. S. et al. Apple SEPALLATA1/2-like genes control fruit flesh development and ripening. Plant J. 73, 1044-1056 (2013).

3. Zhou, K. et al. MdUGT88F1-mediated phloridzin biosynthesis regulates apple development and Valsa canker resistance. Plant Physiol. 180, 2290-2305 (2019).

4. Chagné, D. et al. An ancient duplication of apple MYB transcription factors is responsible for novel red fruit-flesh phenotypes. Plant Physiol. 161, 225-239 (2013).

5. Li, Y. Y. et al. MdCOP1 ubiquitin E3 ligases interact with MdMYB1 to regulate light-induced anthocyanin biosynthesis and red fruit coloration in apple. Plant Physiol. 160, 1011-1022 (2012).

6. Hu, D. G. et al. MdMYB1 regulates anthocyanin and malate accumulation by directly facilitating their transport into vacuoles in apples. Plant Physiol. 170, 1315-1330 (2016)

7. Zhu, L. et al. MdERDL6-mediated glucose efflux to the cytosol promotes sugar accumulation in the vacuole through up-regulating TSTs in apple and tomato. Proc. Natl Acad. Sci. USA 118, e2022788118 (2021).

8. Wang, N. et al. MYB12 and MYB22 play essential roles in proanthocyanidin and flavonol synthesis in red-fleshed apple (Malus sieversii f. niedzwetzkyana). Plant J. 90, 276-292 (2017).

9. Li, Y. et al. Interactions of apple and the Alternaria alternate apple pathotype. Crit. Rev. Plant Sci. 32, 141-150 (2013).

10. Meng, D. et al. Sorbitol modulates resistance to Alternaria alternata by regulating the expression of an NLR resistance gene in apple. Plant Cell 30, 1562-1581 (2018)

11. He, X. et al. Activation of disease resistance against Botryosphaeria dothidea by downregulating the expression of MdSYP121 in apple. Hort. Res. 5, 24 (2018).

12. Gessler, C. \& Pertot, H. Vf scab resistance of Malus. Trees 26, 95-108 (2012).

13. Li, C. et al. A sugar transporter takes up both hexose and sucrose for sorbitolmodulated in vitro pollen tube growth in apple. Plant Cell 32, 449-469 (2020).

14. Meng, D. et al. Apple MdABCF assists in the transportation of S-RNase into pollen tubes. Plant J. 78, 990-1002 (2014).
15. Spolaore, S., Trainotti, L. \& Casadoro, G. A simple protocol for transient gene expression in ripe fleshy fruit mediated by, Agrobacterium. J. Exp. Bot. 52 845-850 (2001).

16. He, P., Shan, L. \& Sheen, J. in Methods in Molecular Biology, Vol. 354 (ed Ronald, P. C.) Ch. 1 (Humana, 2010).

17. $\mathrm{Ma}, \mathrm{X}$. et al. Ligand-induced monoubiquitination of BIK1 regulates plant immunity. Nature 581, 199-203 (2020).

18. Zhang, X. C., Millet, Y. A., Cheng, Z., Bush, J. \& Ausubel, F. M. Jasmonate signalling in Arabidopsis involves SGT1b-HSP70-HSP90 chaperone complexes. Nat. Plants 1, 15049 (2015).

19. Fu, L. et al. The TOR-EIN2 axis mediates nuclear signalling to modulate plant growth. Nature 591, 1-5 (2021).

20. Zhou, Y. et al. Telobox motifs recruit CLF/SWN-PRC2 for H3K27me3 deposition via TRB factors in. Arabidopsis. Nat. Genet. 50, 638-644 (2018).

21. Zhang, N. et al. Engineering artificial microRNAs for multiplex gene silencing and simplified transgenic screen. Plant Physiol. 178, 989-1001 (2018).

22. Wu, S. et al. Establishment of a PEG-mediated protoplast transformation system based on DNA and CRISPR/Cas9 ribonucleoprotein complexes for banana. BMC Plant Biol. 20, 425 (2020).

23. Osakabe, Y. et al. CRISPR-Cas9-mediated genome editing in apple and grapevine. Nat. Protoc. 13, 2844-2863 (2018).

24. Marion, J. et al. Systematic analysis of protein subcellular localization and interaction using high-throughput transient transformation of Arabidopsis seedlings. Plant J. 56, 169-179 (2008).

25. Li, T. et al. The jasmonate-activated transcription factor MdMYC2 regulates ETHYLENE RESPONSE FACTOR and ethylene biosynthetic genes to promote ethylene biosynthesis during apple fruit ripening. Plant Cell 29, 1316-1334 (2017).

26. Akagi, A., Dandekar, A. M. \& Stotz, H. U. A resistance of Malus domestica fruit to Botrytis cinerea depends on endogenous ethylene biosynthesis. Phytopath 101, 1311-1321 (2011).

27. Wu, S. et al. Bacterial effector HopF2 suppresses Arabidopsis innate immunity at the plasma membrane. Mol. Plant-Microbe Interact. 24, 585-593 (2011).

28. Tacken, E. et al. The role of ethylene and cold temperature in the regulation of the apple polygalacturonase1 gene and fruit softening. Plant Physiol. 153, 294-305 (2010).

29. Zhou, J. et al. Differential phosphorylation of the transcription factor WRKY33 by the protein kinases CPK5/CPK6 and MPK3/MPK6 cooperatively regulates camalexin biosynthesis in Arabidopsis. Plant Cell 32, 2621-2638 (2020).

30. Asai, T. et al. MAP kinase signalling cascade in Arabidopsis innate immunity. Nature 415, 977-983 (2002).

31. Li, J., An, B. \& Zhang, X. Identification and promoter analysis of some important storage protein genes from wheat (Triticum aestivum L.). Plant Omics J. $\mathbf{5}$, 326-332 (2012).

32. Grefen, C. et al. A ubiquitin-10 promoter-based vector set for fluorescent protein tagging facilitates temporal stability and native protein distribution in transient and stable expression studies. Plant J. 64, 355-365 (2010).

33. Behera, S. et al. Analyses of $\mathrm{Ca}^{2+}$ dynamics using a ubiquitin-10 promoterdriven Yellow Cameleon 3.6 indicator reveal reliable transgene expression and differences in cytoplasmic $\mathrm{Ca}^{2+}$ responses in Arabidopsis and rice (Oryza sativa) roots. N. Phytol. 206, 751-760 (2015).

34. Gao, X. et al. Silencing GhNDR1 and GhMKK2 compromises cotton resistance to Verticillium wilt. Plant J. 66, 293-305 (2011).

35. Zhu, Q. et al. A MAPK cascade downstream of IDA-HAE/HSL2 ligand-receptor pair in lateral root emergence. Nat. Plants 5, 414-423 (2019).

36. Zhang, $X$. et al. Overexpression of Arabidopsis MAP kinase kinase 7 leads to activation of plant basal and systemic acquired resistance. Plant J. $\mathbf{5 2}$ 1066-1079 (2007).

37. Lu, D. et al. A receptor-like cytoplasmic kinase, BIK1, associates with a flagellin receptor complex to initiate plant innate immunity. Proc. Natl Acad. Sci. USA 107, 496-501 (2010).

38. de Oliveira, M. V. et al. Specific control of Arabidopsis BAK1/SERK4-regulated cell death by protein glycosylation. Nat. Plants 2, 15218 (2016).

39. Djami-Tchatchou, A. T. et al. Dual role of auxin in regulating plant defense and bacterial virulence gene expression during Pseudomonas syringae PtoDC3000 pathogenesis. Mol. Plant-Microbe Interact. 33, 1059-1071 (2020).

40. Cui, F. et al. The Pseudomonas syringae type III effector AvrRpt2 promotes pathogen virulence via stimulating Arabidopsis auxin/indole acetic acid protein turnover. Plant Physiol. 162, 1018-1029 (2013). 